\title{
Fábrica de Objetos: Uma Plataforma para Construção de Objetos de Aprendizagem focada na Usabilidade
}

\author{
João Pedro Dewes Guterres ${ }^{1}$, Silvia Maria Wanderley Moraes ${ }^{1}$ \\ ${ }^{1}$ Faculdade de Informática - PUCRS - Porto Alegre - RS - Brasil \\ joao.guterres@acad.pucrs.br, silvia.moraes@pucrs.br
}

\begin{abstract}
This paper describes a platform for development of learning objects called "Fábrica de Objetos". This platform integrates two systems. The first is an authoring system for the building of learning objects. The second is a web system that works as a repository and that allows, in addition to the storage and visualization of learning objects, cataloging them. In an initial study, we realized a subjective evaluation about the usability of the objects generated by the platform. Subsequently, we applied the practical usability testing for teachers and students. The results of these evaluations were satisfactory.

Resumo. Este artigo descreve a plataforma para o desenvolvimento de objetos de aprendizagem chamada Fábrica de Objetos. Essa plataforma integra dois sistemas. O primeiro deles é um sistema de autoria por meio do qual os usuários podem criar seus objetos de aprendizagem. O segundo é um sistema web que funciona como um repositório e que permite, além do armazenamento e a visualização dos objetos de aprendizagem, a catalogação dos mesmos. Em um estudo inicial, a usabilidade dos objetos gerados na plataforma foi avaliada de forma subjetiva. Posteriormente, foram aplicados testes práticos de usabilidade da plataforma para professores e dos objetos para alunos. Os resultados dessas avaliações foram satisfatórios.
\end{abstract}

\section{Introdução}

Os objetos de aprendizagem surgiram nos anos 2000, quando equipes multidisciplinares formadas por professores, projetistas, programadores, dentre outros profissionais, em prol de uma meta comum, buscaram conciliar diferentes saberes para descobrir formas alternativas de transmitir conhecimento aos alunos [Oliveira e Medina 2007].

Não existe uma definição única para os objetos de aprendizagem. Em IEEE (2002), por exemplo, os objetos de aprendizagem são definidos como "qualquer entidade digital ou não digital que possa ser utilizada para aprendizagem, educação ou treinamento". Já Wiley (2000) os define como materiais didáticos disponíveis em meio digital que podem ser acessados simultaneamente por vários usuários. Analisando-se diferentes definições [Mohan e Brooks 2003, Sosteric e Hesemeir 2004], pode-se dizer que os objetos de aprendizagem são materiais educacionais que permitem a interação individual do aluno com conteúdos e exercícios disponíveis em meio digital e que têm como propósito fixar um determinado conteúdo e, ainda, complementar uma situação de aprendizagem. 
A popularização da educação a distância estimulou o desenvolvimento de objetos de aprendizagem e, consequentemente, também de ambientes que permitissem a criação desses objetos. As dificuldades, no entanto, não estão apenas centradas nas funcionalidades que tais ambientes oferecem, mas também na simplicidade do processo de elaboração de um objeto por professores como pouca familiaridade com ferramentas computacionais. Tão importante quanto a fácil criação é o gerenciamento desses objetos de aprendizagem. Esse gerenciamento de pós-criação, nem sempre é oferecido pelo ambiente de desenvolvimento.

Os objetos de aprendizagem produzidos, geralmente, são catalogados pelos seus criadores em repositórios. A utilização de um repositório específico aos objetos facilita a busca do objeto desejado. Para tornar a categorização dos objetos possível, alguns padrões de metadados (dados sobre os dados) são utilizados para associar os objetos a informações que especifiquem corretamente o seu conteúdo. Um exemplo de repositório é o DSpace [DSpace 2013]. Ele é software de código aberto que implementa um acervo digital, utilizado para repositórios de objetos de aprendizagem.

Essa falta de integração entre o ambiente de desenvolvimento e o repositório dos objetos acabando sendo mais um empecilho para usuários leigos em computação. Foram esses problemas que inspiraram a criação da plataforma Fábrica de Objetos. A plataforma integra um sistema de autoria, que permite a elaboração de objetos de aprendizagem, com um sistema web, que possibilita a visualização desses objetos. $\mathrm{O}$ sistema de autoria foi desenvolvido com o intuito de criar objetos com base em templates, o que simplifica o processo de criação dos objetos. Já o sistema web armazena e organiza os objetos produzidos, servindo, assim, como um repositório. A inserção do objeto no repositório é feita diretamente pelo sistema de autoria no momento em que o professor finaliza o processo de criação do seu objeto. A plataforma ainda permite a internacionalização dos objetos, ou seja, a edição de conteúdos e atividades em diferentes línguas, e também a visualização dos objetos a partir de dispositivos móveis.

Inicialmente, foi realizada uma avaliação subjetiva de usabilidade dos objetos gerados na plataforma. Nessa análise foram consideradas as diretivas de avaliação descritas por Silveira e Carneiro (2012). Em um segundo momento, para avaliar a facilidade de criação dos objetos, bem como as funcionalidades da plataforma, na prática, foram realizados testes de usabilidade com 7 professores. Para avaliar a usabilidade para alunos, foi elaborado um objeto de aprendizagem na plataforma e testado por 30 alunos de uma disciplina inicial do curso de Sistema de Informação. Em todas as avaliações realizadas, os resultados foram muito animadores.

Este artigo está organizado em 7 seções. A Seção 2 comenta algumas plataformas para o desenvolvimento de objetos de aprendizagem semelhantes à plataforma proposta. A Seção 3 detalha a arquitetura da plataforma. A Seção 4 descreve de forma sucinta como criar um objeto na plataforma. As Seções 5 e 6 descrevem as avaliações realizadas bem como os resultados obtidos. E, a Seção 7, por fim, apresenta as considerações finais deste trabalho. 


\section{Trabalhos Relacionados}

Para definir a arquitetura da Fábrica de Objetos foram estudadas algumas plataformas para o desenvolvimento de objetos de aprendizagem. Nesse estudo foram consideradas plataformas cuja finalidade fosse especificamente a criação de objetos de aprendizagem e que possuíssem interfaces simples. Foram analisadas as plataformas My Mobile Learning Engine (MyMLE), Mobile Learning Engine Moodle (MLE Moodle) e eLearning XHTML Editor (eXe).

A plataforma MyMLE ${ }^{1}$ [Batista e Mamari 2010] permite a criação de objetos de aprendizagem exclusivamente para celulares com plataforma Java. Permite também a inclusão de diferentes tipos de questionários, além da inclusão de som, vídeo e conteúdos em HTML. A ferramenta se mostra muito interessante para criar objetos de aprendizagem, porém é limitada apenas a celulares e não possui flexibilidade de layout para diferentes tipos de cenários. Como MyMLE foi criado em 2009, antes da popularização do uso de smartphones, seu escopo de criação de objetos é restrito a poucos recursos audiovisuais, tendo em vista a limitação de recursos nos celulares. Além disso, a ferramenta não permite a internacionalização dos conteúdos do objeto em várias línguas.

A plataforma $\mathrm{MLE}^{2}$ Moodle [Ribeiro e Medina 2009] é um plugin do Moodle ${ }^{3}$ de código fonte livre para desenvolvimento de objetos de aprendizagem para web e celulares com plataforma Java (J2ME). Sua diferença para o MyMLE é a possibilidade de acessar os conteúdos via internet a partir do Moodle. A ferramenta possui mais acessibilidade, pois permite a visualização dos objetos em diferentes dispositivos, entretanto possui os mesmos problemas de flexibilidade de layout, internacionalização dos conteúdos e recursos audiovisuais do MyMLE.

A plataforma eXe ${ }^{4}$ [Azevedo 2008] permite criar objetos de aprendizagem e exportá-los utilizando XML e HTML, seguindo o padrão SCORM 1.2. A plataforma possui uma abordagem muito interessante para criação objetos de aprendizagem. Ela cria páginas a partir de layouts prontos e as exporta diretamente para páginas web. Entretanto, o design da sua interface é limitado e não permite a internacionalização do conteúdo para várias línguas. Outro fator a ser considerado é a descentralização de seu conteúdo, que está em arquivos XML e HTML.

Na seção seguinte é descrita a arquitetura da plataforma Fábrica de Objetos.

\section{Arquitetura da Plataforma}

A plataforma Fábrica de Objetos foi implementada na linguagem Java e em PHP. Ela segue o padrão de projeto MVC [Reese 2003], sendo que a camada de dados é responsável pela comunicação com um banco de dados MySQL 5. O sistema de autoria foi desenvolvido em Java e o sistema web, em PHP.

\footnotetext{
${ }^{1}$ http://mle.sourceforge.net/mymle/

${ }^{2}$ http://mle.sourceforge.net/mlemoodle/index.php?lang=en

${ }^{3}$ https://moodle.org/

${ }^{4}$ http://exelearning.org/
} 
O sistema web é responsável pela comunicação com o banco de dados e exibição dos objetos para os alunos. A linguagem HTML5 foi escolhida para estruturação e apresentação de conteúdo, pois inclui novas funcionalidades como semântica e acessibilidade. Os aspectos de design do layout web são reproduzidos a partir da linguagem CSS3 que gerencia a apresentação dos objetos com base em propriedades incluídas no banco de dados. A interatividade e usabilidade do objeto de aprendizagem é gerenciada pelo jQuery e pela biblioteca reveal.js. A integração do sistema de autoria com o sistema web, bem como as tecnologias usadas podem ser vistas na Figura 1.

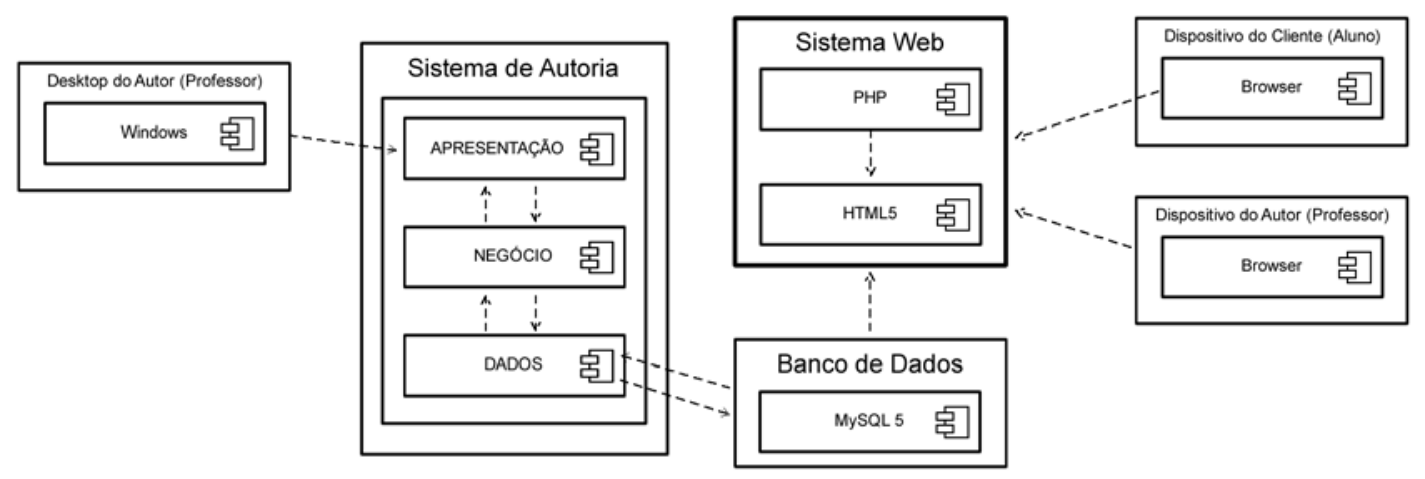

Figura 1. Estrutura da plataforma Fábrica de Objetos.

Nas seções seguintes, os sistemas de autoria e web são detalhados.

\subsection{Sistema de Autoria}

A criação de um objeto no sistema de autoria inicia com a inclusão dos metadados desse objeto. É a partir desses metadados (descrição, área, autor,...) que o sistema web posteriormente organizará os objetos no repositório. São de responsabilidade do sistema de autoria a criação, alteração e remoção tanto de objetos quanto das páginas que os compõem. Esse sistema ainda permite a duplicação de objetos existentes, o que facilita a internacionalização dos conteúdos, possibilitando a adaptação de um objeto para outra língua. Cabe mencionar que o sistema de autoria permite a edição de objetos mesmo após a sua finalização (indicação que está pronto para ser exportado para o sistema web).

Para criar uma página em um objeto, é necessário, primeiramente, escolher um template predefinido para diagramar os conteúdos dessa página. Há templates para conteúdos e para exercícios, como mostra a Figura 2. A plataforma, atualmente, possui 11 templates para conteúdos e 2 templates para exercícios. Os templates de conteúdo oferecem diferentes diagramações para textos, imagens e até mesmo para código-fonte. Já os templates de exercícios são ambos de múltipla escolha, variando a forma como o feedback é apresentado ao aluno. Em um deles, há um feedback para cada alternativa, e no outro, há apenas dois feedbacks: um para resposta certa e outro para as demais respostas. Cabe mencionar que a estrutura interna definida para os templates foi pensada visando já a expansão dos mesmos. Portanto, a quantidade atual de templates não é de fato um limitador. Além disso, o uso de templates para criação de páginas, permitiu separar toda a estrutura visual (HTML5, CSS3 e jQuery) dos conteúdos da página, o que melhora muito tanto a construção quanto a manutenção das páginas dos objetos. A Figura 3 ilustra a estrutura da página dos objetos. 

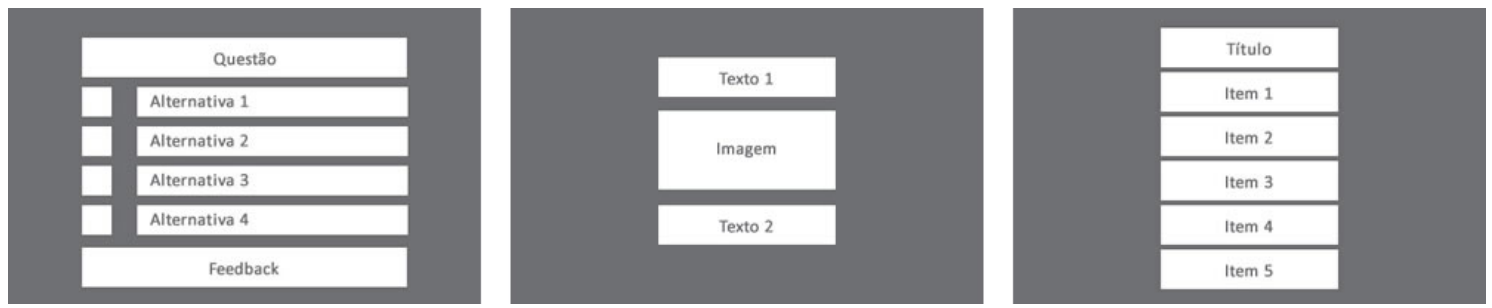

Figura 2. Exemplos de Templates.

Para organizar uma página de exercícios e viabilizar a avaliação (contendo o desempenho dos alunos nos exercícios e retornando um feedback de acordo com seu desempenho), foi necessário agrupar as páginas de exercícios em uma estrutura chamada módulo de exercícios. Cada módulo é tratado como uma página do objeto e deve ter no mínimo um exercício. É possível criar vários módulos de exercícios em um único objeto.

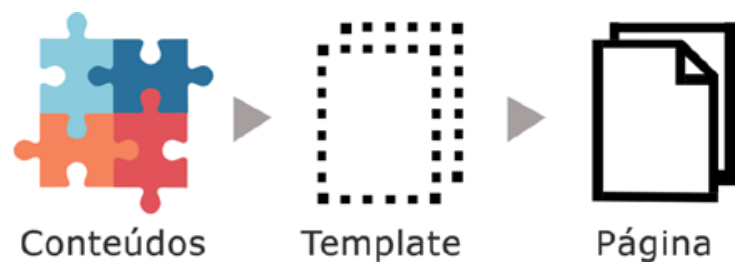

Figura 3. Estrutura das páginas que compõem os objetos de aprendizagem.

\subsection{Sistema web}

O sistema web permite gerenciar os objetos de aprendizagem, funcionando como um repositório. A Figura 4 ilustra a organização dos objetos no repositório e a estrutura interna desses objetos (compostos de metadados e páginas).
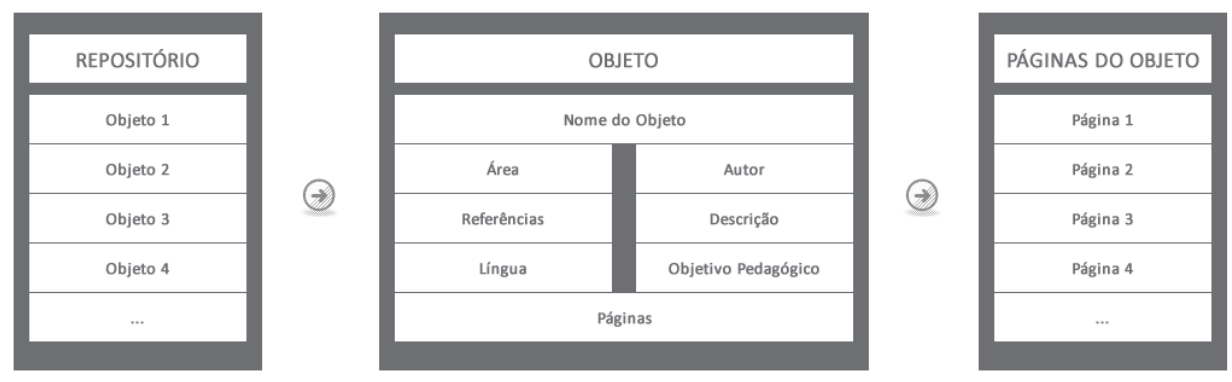

Figura 4. Estrutura do objeto de aprendizagem.

Todos os objetos criados são salvos em um banco de dados e podem ser acessados tanto pelo sistema de autoria quanto pelo sistema web. A principal característica desse repositório online é a capacidade de exibir dinamicamente em tempo de execução o objeto atualizado a partir do registro no banco de dados. Os objetos estão catalogados nesse repositório de acordo com os seus metadados.

No objeto de aprendizagem, a navegação principal é feita horizontalmente, acessando as páginas de conteúdos. Entretanto, para acessar os exercícios a navegação se torna vertical, retomando a ideia de um conjunto de exercícios agrupados. A Figura 5 ilustra a estrutura de navegação dos conteúdos e dos módulos de exercícios. 


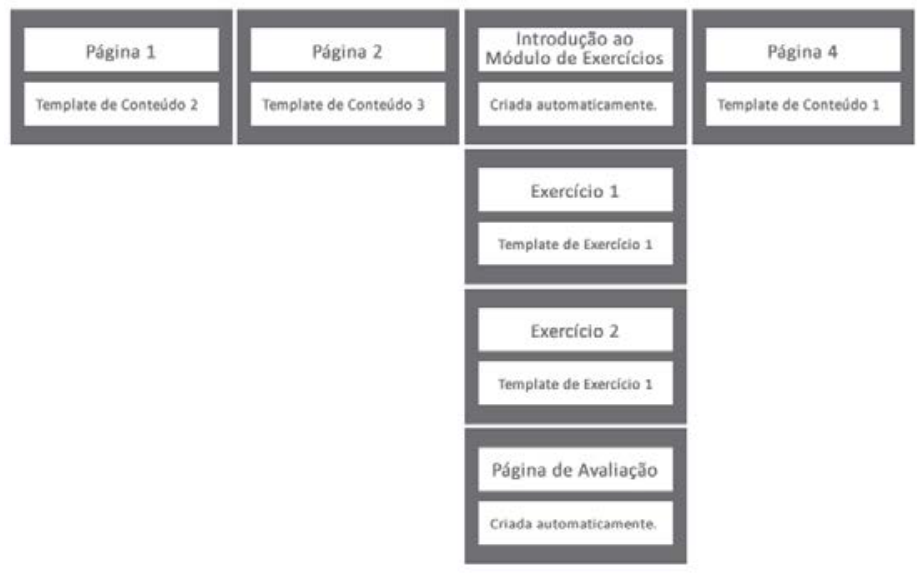

Figura 5. Forma de navegação dos objetos.

A plataforma possui dois tipos de feedbacks: um feedback imediato e uma página de avaliação. O feedback imediato tem como característica exibir a correção do exercício e uma breve explicação sobre as razões de uma resposta estar incorreta ou correta [Nogueira 2006]. Já a página de avaliação tem como característica apresentar uma indicação (ou links) a materiais. Ambos são editados no sistema de autoria. plataforma.

A próxima seção descreve de forma resumida como criar um objeto na

\section{Processo de Criação de um Objeto de Aprendizagem}

A primeira etapa para a construção de um novo objeto de aprendizagem é acessar a opção 'Criar' no menu 'Objeto' do sistema de autoria Assim, o autor poderá definir um objeto desde o início, colocando as informações básicas (metadados) e construindo suas páginas. Outra forma de criar um objeto é duplicar um objeto já existente no banco de dados e editar seus conteúdos. A partir da criação do objeto, o autor tem acesso à tela de criação de páginas. É possível, então, escolher o tipo de página que se deseja criar (página com conteúdo ou exercícios). A Figura 6 mostra a interface da plataforma através da qual o autor visualiza o objeto aberto, bem como as opções de cadastro das páginas.

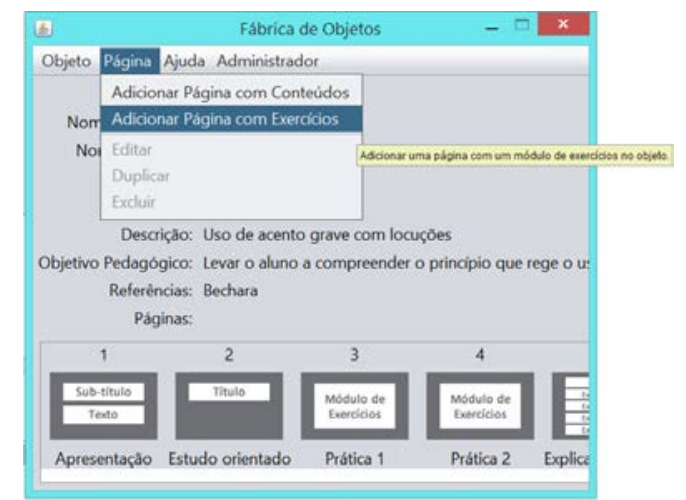

Figura 6. Cadastrando páginas no objeto. 
Na página principal, o autor consegue criar, editar, duplicar, remover e alterar a ordem das páginas do objeto. Após a criação das páginas, é possível salvar o objeto e concluí-lo em outro momento ou liberar o acesso dos alunos ao objeto, finalizando-o e tornando-o disponível para acesso no sistema web. A Figura 7 apresenta a sequência de telas da interface web onde o usuário escolhe um objeto (1), visualiza seus metadados (2) e acessa o objeto (3).

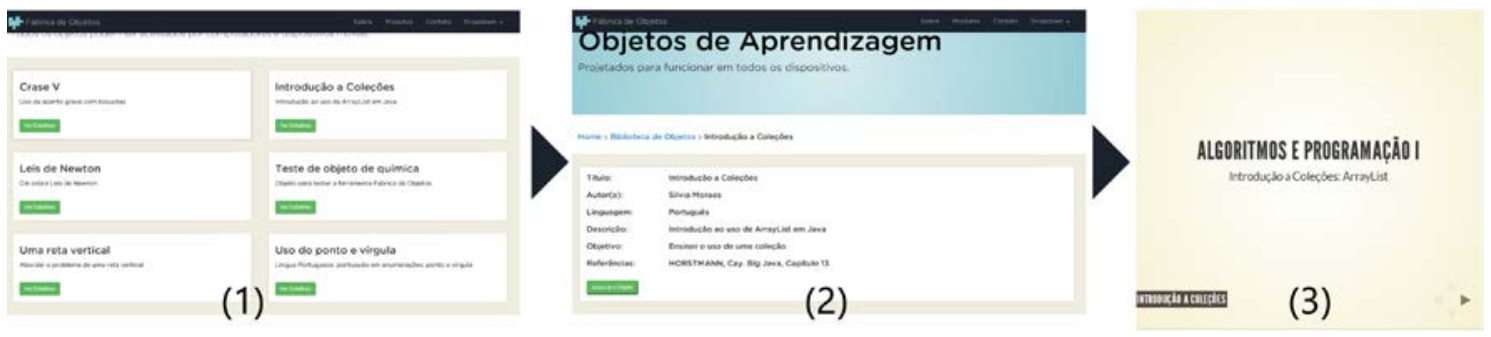

Figura 7. Sistema web da plataforma Fábrica de Objetos.

A interface possibilita a livre navegação no objeto de aprendizagem, permitindo ao usuário navegar tanto pela seta do teclado quanto pelos ícones na área inferior à direita. Além da navegação pela seta, o usuário pode visualizar um menu padrão ao clicar na tecla "esc" do teclado.

\section{Avaliação Subjetiva de Usabilidade dos Objetos de Aprendizagem}

Segundo Silveira e Carneiro (2012), há sete condições essenciais que devem ser consideradas ao se avaliar a usabilidade de um objeto de aprendizagem. Essas condições, por sua vez, foram subdivididas e descritas através de diretrizes. Neste estudo preliminar, foi analisado o potencial da plataforma quanto à geração de objetos de aprendizagem que atendem às diretivas de cada condição relativa à usabilidade. $\mathrm{O}$ quadro 1 resume o resultado dessa análise. Esse quadro indica se as condições essenciais encontradas nos objetos produzidos através da plataforma são atendidas plenamente, parcialmente ou não atendidas. Mesmo para as condições atendidas parcialmente, os objetos feitos na plataforma satisfazem em torno de $90 \%$ das diretivas da referida condição.

\section{Quadro 1. Avaliação subjetiva dos objetos gerados na plataforma}

\begin{tabular}{|l|c|c|c|}
\hline Condição & Plenamente & Parcialmente & Não Atende \\
\hline $\begin{array}{l}\text { "Explicitar claramente um } \\
\text { objetivo pedagógico" }\end{array}$ & & $\mathrm{X}$ & \\
\hline "Priorizar o digital” & & $\mathrm{X}$ & \\
\hline "Prover auxílio aos usuários” & $\mathrm{X}$ & & \\
\hline "Proporcionar interatividade” & $\mathrm{X}$ & & \\
\hline "Proporcionar interação" & & & $\mathrm{X}$ \\
\hline "Fornecer feedback constante” & $\mathrm{X}$ & & \\
\hline "Ser autocontido" & $\mathrm{X}$ & & \\
\hline
\end{tabular}


Na condição “Explicitar claramente um objetivo pedagógico”, apenas a diretriz referente à disponibilização de materiais complementares ao objeto não foi implementada. Já na condição "Priorizar o digital”, não foram atendidas as diretivas referentes à criação de um mecanismo que alerte o usuário caso seu browser não funcione corretamente e, ainda, a diretiva referente à explicitação de quais softwares são necessários para executar os objetos.

A única condição que de fato não foi implementada diz respeito a "Proporcionar interação”, ou seja, permitir a interação entre alunos e usuários nos objetos de aprendizagem. A versão atual da plataforma não permite que os resultados avaliativos gerados pelos objetos sejam compartilhados. O compartilhamento dessas informações possibilita que vários professores discutam o desempenho dos alunos.

Todas as diretivas não atendidas podem ser incorporadas à plataforma, no futuro.

\section{Testes de Usabilidade}

Nesta seção descrevem-se os testes de usabilidade aplicados tanto em professores quanto em alunos e também os resultados obtidos.

\subsection{Teste com Professores}

Este teste foi realizado com 7 professores que elaboram ou já elaboraram objetos de aprendizagem. O objetivo do teste era a criação de um pequeno objeto de aprendizagem utilizando os recursos disponíveis da plataforma Fábrica de Objetos. Este teste visava identificar se a plataforma atendia a seu propósito, se a mesma era útil e de fácil uso pelos professores.

Foram utilizados como instrumentos de coletas de dados 2 questionários aplicados de forma presencial e seguindo um roteiro pré-estabelecido, o qual é enumerado a seguir:

1. Aplicação do questionário pré-teste, contendo a identificação do professor e alguns dados sobre sua experiência na elaboração de objetos de aprendizagem;

2. Construção do objeto na plataforma;

3. Preenchimento do questionário pós-teste, contendo 4 questões objetivas e 3 questões dissertativas sobre a experiência de construir um objeto de aprendizagem na Fábrica de Objetos.

Dos professores que testaram a plataforma, apenas um deles se considerou um usuário avançado, os outros se consideraram usuários intermediários de computadores. Em média eles possuem 3,2 anos de experiência na criação de objetos. Esses professores somados elaboraram aproximadamente 61 objetos de aprendizagem.

Analisando-se as respostas do questionário pós-teste, percebeu-se que 5 professores acharam fácil construir um objeto e 2 acharam muito fácil. Todos conseguiram criar um pequeno objeto e acharam clara a sequência de passos para criar um objeto na plataforma. Os professores que acharam muito fácil criar um objeto não necessitaram de qualquer auxilio para interagir com a plataforma durante o teste. 
Os objetos gerados na plataforma atingiram as expectativas de todos os professores. Os professores que realizaram o teste afirmaram que incorporariam a plataforma ao seu conjunto de ferramentas para produção de seus objetos de aprendizagem. Em geral, os aspectos apontados para melhorar a plataforma foram a inserção de novos recursos, como por exemplo: novos templates e a captura das estatísticas do desempenho dos alunos nos exercícios.

\subsection{Teste dos Objetos de Aprendizagem com Alunos}

Este teste foi realizado no semestre de 2014/1 com 30 alunos das turmas 168 e 178 da disciplina de Algoritmos e Programação I, do curso de Sistemas de Informação. O objetivo do teste foi verificar a usabilidade dos objetos de aprendizagem criados na plataforma. Além disso, analisar se o objeto favoreceu à aprendizagem do conteúdo e se o aluno recomendaria o objeto para um colega. O objeto utilizado foi "Introdução a Coleções em Java”, criado pela professora da disciplina.

Nesta avaliação, foi utilizado como instrumento de coleta de dados: um questionário online, contendo o nome do aluno, 4 questões objetivas e um campo para sugestões sobre o objeto criado na Fábrica de Objetos. O questionário foi aplicado de forma online através do Sistema Qualtrics ${ }^{5}$ - um serviço de organização e coleta de dados para pesquisa via web. O questionário foi aplicado após o uso do objeto de aprendizagem.

Dos alunos que testaram a plataforma, 12 deles (40\% do total) não conheciam os objetos de aprendizagem antes do teste. Segundo a avaliação, 97\% dos alunos apontaram que a apresentação dos conteúdos em um objeto de aprendizagem favoreceu a aprendizagem do tema. Em termos de usabilidade, 87\% dos alunos acharam fácil navegar no objeto de aprendizagem. Com a utilização do objeto, 93\% dos alunos recomendariam o objeto para o ensino de coleções. No campo referente a sugestões, os alunos elogiaram o objeto e indicaram algumas melhorias.

\section{Considerações Finais}

Este artigo descreveu a plataforma Fábrica de Objetos, a qual possui entre suas principais contribuições quanto ao desenvolvimento de objetos: uma interface simples, possibilitando a construção de objetos de aprendizagem mesmo por usuários com pouco conhecimento sobre ferramentas computacionais. Além de produtores de objetos, os usuários podem atuar como gestores de conteúdos, podendo realizar atualizações nos conteúdos e exercícios e replicar objetos em outras línguas.

Através da integração de várias etapas envolvidas no processo de criação é possível organizar os objetos em um repositório e visualizá-los em dispositivos móveis. Embora a plataforma ainda precise ser aprimorada e mais testes precisem também ser realizados, acredita-se, com base nas avaliações feitas, que os objetos gerados pela ferramenta atendem a maioria dos critérios de usabilidade considerados.

\footnotetext{
${ }^{5}$ http://www.qualtrics.com/
} 


\section{Referências Bibliográficas}

AZEVEDO, Susana. Tutorial eXe eLearning XHTML editor (versão 1.0x), 2008.

BATISTA, Silvia Cristina Freitas; MAMARI, Alex Botelho. MyMLE - MOODLE Ferramenta para desenvolvimento de Objetos de Aprendizagem para Celulares, 2010. Disponível por www em: http://plataforma.nie.iff.edu.br/projetomlearning/arquivos/Tutorial\%20MyMLE.pdf

DSPACE. DSpace 4.x Documentation, 2013.

IEEE. Draft Standard for Learning Object Metadata: IEEE 1484.12.1-2002, 2002.

MOHAN, Permanand; BROOKS, Christopher. Engineering a Future for Web-based Learning Objects. ICWE, volume 2722 of Lecture Notes in Computer Science, page 120-123. Springer, 2003.

NOGUEIRA, Rommel Soares Ferreira. Efeitos do tempo de exposição ao feedback na aprendizagem em treinamento baseado na web, Dissertação (Mestrado em Psicologia)-Universidade de Brasília, Brasília, 2006.

OLIVEIRA, Leandro Ramos de; MEDINA, Roseclea Duarte. Desenvolvimento de objetos de aprendizagem para dispositivos móveis: uma nova abordagem que contribui para a educação, 2007. Disponível por www em: http://www.cinted.ufrgs.br/ciclo9/artigos/4aLeandro.pdf

REESE, George. Java Database Best Practices, 2003.

RIBEIRO, Patric da Silva; MEDINA, Roseclea Duarte. Mobile Learning Engine Moodle (MLE - Moodle): das funcionalidades a validação em curso a distância utilizando dispositivos móveis, 2009. Disponível por www em: http://seer.ufrgs.br/index.php/renote/article/view/14026/7915

SILVEIRA, Milene Selbach; CARNEIRO, Mára Lúcia Fernandes. Diretrizes para a Avaliação da Usabilidade de Objetos de Aprendizagem. In: XXIII Simpósio Brasileiro de Informática na Educação (SBIE 2012)

SOSTERIC, Mike; HESEMEIER, Susan. A first step towards to a theory of learning objects. In Online Education using Learning Objects, pages 32-42. RoutledgeFalmer, 2004.

WILEY, David A., Connecting learning objects to instructional design theory:A definition, a metaphor, and a taxonomy, 2002. 